\title{
A TAXONOMICAL FRAMEWORK OF SOCIO-CULTURAL HAZARDS IN TRANSPORT HUBS
}

\author{
SIMONE ROZZI ${ }^{1}$, ALESSIA GOLFETTI $^{1}$, MICHAEL MINKOV $^{2}$, MARK ROBINSON ${ }^{3}$, \\ FERHAN ŞENGÜR ${ }^{4} \&$ CARLO DAMBRA ${ }^{5}$ \\ ${ }^{1}$ Deep Blue s.r.l., Rome, Italy. \\ ${ }^{2}$ Varna University of Management, Varna, Bulgaria. \\ ${ }^{3}$ University of Leeds, Leeds, UK. \\ ${ }^{4}$ Anadolu University, Eskisehir, Turkey. \\ ${ }^{5}$ Proprs, Ltd, Oxford, UK.
}

\begin{abstract}
This article presents a taxonomical framework that supports the considerations of socio-cultural hazards that may affect crowd management in transport hubs, i.e. airports, ports, underground and train stations, both in normal and emergency situations. Such hazards include communication breakdowns with passengers due, for instance, to language barriers; increased potential for revolts, as in stranded passenger situations; misreporting of security threats; and uncooperative behaviour in case of emergencies. Such socio-cultural hazards are not normally considered from the integrated perspective of transport hub operators, e.g. security staff, first responders and service assistants as well as safety and security managers. The present study provides an integrated perspective of these hazards as a means to increase the performance of transport staff members that interact with the public and with passengers on a daily basis. The methodology used to develop the framework comprises: (i) a focus group with relevant experts, (ii) semi-structured interviews at operational facilities with front-end practitioners, and (iii) a review of academic literature and media reports. The framework has also been qualitatively corroborated with transport operators in dedicated interviews and a focus group session. The study identified 10 socio-cultural hazards that were combined into a single framework comprising three high-level sub-categories: (i) crowd-staff interactions, (ii) crowd-crowd interactions, and (iii) crowd-environment interactions. The framework of socio-cultural factors can increase staff's awareness of relevant socio-cultural hazards, their potential consequences in both normal and emergency situations, and the associated mitigation strategies. In turn, this can increase the quality and continuity of service, safety and security in the management of members of the public and passengers in transport hubs.
\end{abstract}

Keywords: crowd management, disruptive passenger behaviour, emergency management, emergency preparedness and training, risk and security, socio-cultural hazards, transport hubs.

\section{INTRODUCTION}

The effective and safe management of the travelling public is an important consideration for front-end operators of airports, ports, railways and underground stations. The operators of such transport hubs include service personnel (e.g. desk information officers, ticket collectors), security staff (e.g. police, security guards and screeners), and first responders (e.g. fire fighters, medical personnel). Collectively, these operators are responsible for monitoring, informing, assisting and instructing an enormous flow of passengers of different ages, nationalities and cultures, both in normal and emergency situations (e.g. immediate or controlled evacuations, stranded passenger scenarios, etc.).

However, ensuring the smooth, secure and safe movement of passengers is not easy. Operators need to be able to address a plethora of needs and expectations of different passengers and crowds, who may also have linguistic difficulties, and, depending on the situation, may exhibit strong distress, fear or anger. In particular, transport hub operators need to be able to address successfully the variety of socio-cultural hazards ( $\mathrm{SCHs}$ ) that may arise in the transport hub. 
SCHs correspond to behaviours of transport hub users and staff that are dysfunctional to the effective, secure and safe transit of the public in the hub. Such hazards may include communication breakdowns among passengers and staff due to, for instance, language barriers; increased potential for revolts, as in stranded passenger situations; misreporting of security threats; as well as uncooperative behaviour in case of emergencies. As their name suggests, SCHs have both social and cultural determinants, such as age, gender, education level, social status and cultural background. Managing these kinds of behavioural hazards is part of the operational demand of front-end operators of transport hubs. Such hazards may be a source of stress and discomfort for both staff and passengers, potentially reducing passenger satisfaction at a given hub. Furthermore, if not adequately mitigated, SCHs may lead to interruptions/slowing down of passenger throughput, potential for injury, (safety) equipment damage, reputational damage for relevant transport organisations, and potential for legal litigation.

SCHs may occur both in normal situations and emergencies. In the former case, SCHs may be the triggers themselves of the emergency. This is the situation that may arise, for instance, when angry passengers stuck in a transport terminal, exasperated by a long delay following a service disruption, behave aggressively with staff, or when passengers of two different cultural groups located in the same terminal start to argue with each other. Although not necessarily triggering an immediate health and safety risk, these situations require an adequate intervention by staff in order to ensure/restore continuity of service (i.e., flow of passengers in transit) and the security of the involved staff and the other passengers.

In the case of ongoing emergencies-e.g., evacuations, stranded passengers following transport service disruptions-SCHs may aggravate emergency consequences. SCHs can in fact create the potential for more serious consequences than would have occurred in the absence of the SCHs concerned. For instance, communication breakdowns between staff members and the public due to language differences may significantly slow down evacuation time, and may result in inadvertent discrimination towards some sociocultural groups of passengers (e.g., less educated, non-English speaking foreigners), which in turn may be more likely to suffer higher injuries and casualties. Similarly, the failure of passengers to comply with a request to remain in a confined environment during an ongoing emergency (e.g., technical failure) may result in passengers leaving the assigned area and exposing themselves to health and safety hazards. Both situations may result in higher potential for injury and casualties than if the specific SCH not materialised.

The notion of SCHs overlaps with, but is not limited to, the notion of disruptive passenger behaviour. This definition captures instances in which passengers may fail to respect good rules of conduct (for instance based on staff's instructions), may disrupt good order and discipline of operation, and may create a potential safety or security threat [1-3]. Examples of disruptive passenger behaviour include, for instance, consumption of narcotics, refusal to comply with instructions, sexual abuse, and making threats of any kind towards the crew, other passengers and staff [3]. Often associated with alcohol consumption, disruptive passenger behaviour has been especially highlighted in relation to in-flight service, during which cabin staff cannot count on external resources (e.g. help from police forces), but have to deal with the passenger(s) concerned, at least until the aircraft is on the ground. Cheng-Hua and Hsin-Li [4] have however observed that ground service staff should also be prepared to identify and mitigate such disruptive behaviours, as their occurrence on the ground (e.g. airport terminal) is a predictor of their occurrence in the air. Also, incidents linked to disruptive behaviour incidents may involve verbal or physical violence. For instance, Hella [5] provides a diagrammatic representation showing how passenger behaviour may move from the initial 
triggering phase (e.g. luggage dispute, mistreatment by staff), to the following phases of escalation, aggression (verbal and/or physical), and return to normal, baseline behaviour. The ICAO Doc 9811 [6] adopts a four-level scheme to classify the seriousness of unruly/disruptive passenger behaviour, which ranges from verbally disruptive behaviour (level 1) and physical abusive behaviour (level 2), to life-threatening behaviour (level 3) and breach of flight crew compartment (level 4).

Implicit in the definition of disruptive passenger behaviour is a focus on misconduct, potential for violence and criminal offence, and the need for staff to respond with adequate communication and, especially, physical containment actions (e.g. handcuffing). While being highly functional to the safety and security of the transport industry, this characterisation fails, however, to account for (minor) incidents in which the passenger involved does not necessarily intend to create a problem, and in which socio-cultural factors should be considered - such as in situations involving first-time fliers, or passengers of other nations or culture failing to comply with local security regulations.

Indeed, the importance of socio-cultural factors in crisis management has been highlighted in studies of natural disasters such as tsunami, earthquakes, hurricanes and floods [7]. In particular, such studies highlighted that culture can act both as an enabler of and a barrier to effective disaster response. For instance, the positive role of culture towards disaster was observed in relation to the Indian Ocean Tsunami. During that event, some communities had indigenous knowledge about the manifestation of an imminent tsunami, which enabled them to take preventive action (i.e. move away from the sea) and survive, as opposed to migrants and tourists who did not have local knowledge [8]. In particular, the communities were aware of the signs of an imminent Tsunami: unusual behaviours of birds and animals, and low tide. At the same time, culture may play a negative role in effective disaster response. Various cases have been reported in the literature regarding communities that refuse to evacuate a dangerous area - dangerous, for instance, because of the closeness of a volcano - until advised to do so by their local leaders [9]. These instances highlight that cultural background influences the behavioural responses to hazards. These considerations are highly relevant for transport hubs, which usually accommodates large groups of transit passengers of different social and cultural backgrounds, who may react differently to the same instruction, hazard or threat.

With these considerations in mind, the present study aims to investigate the potential SCHs that may occur in transport hubs. In particular, the study provides a preliminary, qualitative, taxonomical framework of SCHs in transport hubs. The goal is to capture the range of SCHs that operators of transport hubs may have to confront in normal and emergency situations. This knowledge base will help transport hub stakeholders in developing appropriate mitigations, such as socio-culturally aware risk management models and training programmes. The study is organised as follows: the Section 2 will describe the methodology; Section 3, the framework; Section 4 will address discussion and conclusions.

\section{METHODOLOGY}

The framework of SCHs presented in this study was developed by adopting an incremental, qualitative, multiple-data collection approach, which is illustrated in the reminder of this section. The data collection comprised the following activities:

- 1-day workshop with experts. This was attended by 21 safety and security experts from the aviation, maritime, railway and metro domains, and coming from the European Union, Turkey and Israel. The workshop took place in November 2015 in Amsterdam. It was 
organised around two main parts. Part one, familiarisation, briefed experts about the research context, aims and objectives, and preliminary results. Part two, data gathering, aimed at collecting expert feedback from participants by means of three group sessions. In group sessions 1 and 2, feedback was collected by means of individual questionnaires and structured group discussions. The questionnaire items covered the areas of security training, risk management practices, communication with passengers, and relevant regulations. The group discussions allowed participating experts to provide further feedback on issues not included in the questionnaire (see below) and to report potential socio-cultural hazards;

- 17 Semi-structured interviews with front-end staff. The interviews were conducted over the period July 2015-April 2016 during site visits to an airport $(n=1)$, a high-speed train company $(n=2)$, and railway police $(n=2)$ facilities in Italy. Personnel interviewed included front-end staff such as stewards of high-speed trains, passengers' assistants, and security screeners and police officers. Each interview lasted between $40 \mathrm{~min}$ and $1 \mathrm{~h}$. They were supported by a 24-item questionnaire organised in four parts: biographical data ( $\mathrm{n}$ of items $=4)$, problematic passenger groups $(n=8)$, current security training $(n=6)$, and management of emergency situations $(n=6)$. The items were open ended to allow for the collection of plenty of feedback.

- Literature and media reviews. Data collection included also a literature review of relevant academic articles and media reports. The review focused on the identification of incidents between service personnel and passengers in transit at transport hubs.

The collected data was analysed qualitatively in a bottom-up fashion, building on the procedures described in $[10,11]$. The first step of the analysis consisted of familiarising the researchers with the data, which consisted of notes, visits reports, audio recordings, and intermediate research reports. Then, this data corpus was reviewed in order to identify initial, preliminary categories of SCHs that emerged from the interviews, the focus group, and the literature review respectively. This process involved numerous judgements about the meaning and the significance of the data examined, and the potential categories of SCHs discussed. Subsequently, the preliminary categories of SCHs were systematically compared with each other. This comparison led to some of the SCHs being either refined and/or aggregated according to similarity into more abstract, generalisable categories of SCHs, or discarded because they were insufficiently substantiated by the available evidence. Subsequently, the SCHs identified were subjected to two internal revisions in which the research team qualitatively checked them for overlaps, completeness, accuracy, and relevance. This process led to an initial definition of a total of 17 SCHs.

The initial version of the ECCS framework was subjected to a corroboration cycle in which feedback was collected by three operational experts and one safety manager of two Turkish airports. While this revision indicated that the framework captured relevant categories of $\mathrm{SCHs}$, it was also instrumental in spotting similar categories that could be further aggregated. This triggered a further revision of the framework by the research team, which led to the final form of the framework: a total of 10 categories of SCHs grouped in three macro categories (as will be described in the next section).

\section{RESULTS: THE ECCS FRAMEWORK}

The SCHs are presented in the context of the Environment, Crowd, other Crowd members and Staff (ECCS) framework. ECCS assumes that to understand the SCHs that can arise from a multicultural crowd, it is necessary to consider the surrounding context in which the crowd 
is located. SCHs in transport hubs lie at the interaction between the crowd (C) and the components of the surrounding context. Such components include:

- $\operatorname{Staff}(\mathrm{S})$, i.e. the operators that can be found at the front end of large transport hubs. These include roles involved in normal day-to-day operations (e.g. screeners, information desk assistants, police officers) and first responders (medics, fire-fighters, etc.);

- Other crowd members $(\mathrm{C})$, i.e. the other crowd members that a person or a group can interact with in a transport hub. Also, interaction with other crowd members may trigger some types of cultural-dependent hazardous behaviours, hence why the $\mathrm{C}$ component appears twice in the model;

- The environment (E), i.e. the information and the physical space that can be found in the transport hub. The public's experience of transport hub includes the gathering of information from various sources, such as aural messages (audio alarms; public messages); digital and physical signage; SMS; social-media (Facebook, etc.). Furthermore, passengers have to move across the hub layout and may interact with various types of equipment.

It is in the interactions between the pivotal components of the framework - i.e. crowd members, and the surrounding contextual components - that one should look at to see the occurrence of socio-cultural hazards. SCHs can be found in the staff-crowd interaction; crowd-crowd interaction (also called within-crowd interaction); and crowd-environment interaction (see Fig. 1). Thus, the ECCS model identifies the interactions between the crowd and the different contextual components that may lead to incidents and service interruptions in a transport hub. The model and its components are shown in Fig. 1. The pivotal element of the model is the crowd, around which the other contextual elements are found. The next sections describe the SCHs that can be found in these interactions.

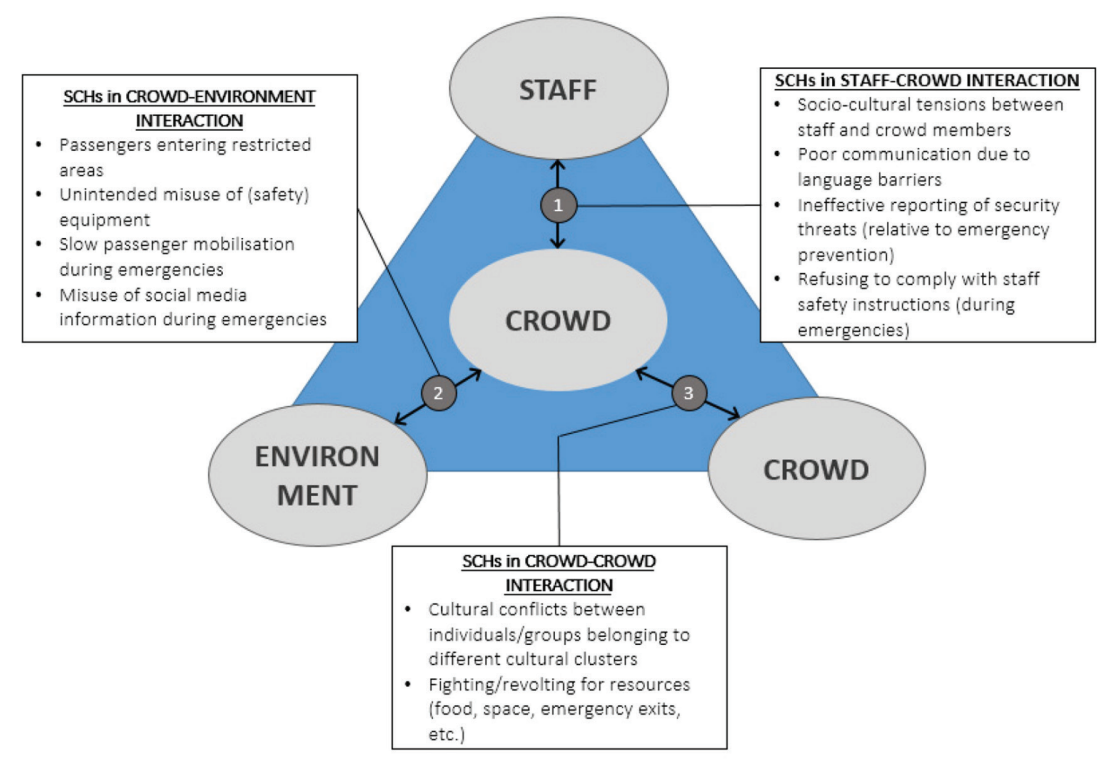

Figure 1: The ECCS model and its components (numbers identify the interactions of the model). 


\subsection{Crowd-staff interactions}

\subsubsection{Socio-cultural tensions between staff and crowd members}

At least three types of socio-cultural tensions may occur between staff and passengers in transport hubs. First, staff may fail to account for the cultural diversity of the passengers they have to deal with. In particular, they may fail to provide equal (health care/emergency) assistance/treatment to passengers of different cultures (especially ethnical or racial minorities) because of stereotypes and prejudice. Stereotyping can be defined as 'the process by which people use social categories (e.g. race, sex) in acquiring, processing, and recalling information about others' [12]. In general, individuals are frequently not aware of the activation or the impact of stereotyping on their perceptions, emotions and behaviour. Stereotype-linked bias is an automatic and unconscious process, and can occur even among persons who are not outwardly prejudiced [13,14]. To Jones [15] stereotyped responses of staff can occur especially in situations characterised by intense time pressure, resource constraints, uncertainty and high/cognitive demand, such as emergencies. Ultimately, stereotyping may result in staff discriminating against some cultural groups when providing assistance (for instance because of religious differences).

Second, staff may inadvertently engage in behaviours considered unacceptable/offensive from the perspective of a passenger of another culture [16]. For instance, tensions may arise due to staff improperly handling sacred/personal items at security checks; staff using inadequate body language (e.g. a male security officer talking directly to a Muslim woman while ignoring her husband); staff making requests considered unacceptable by other cultures (e.g. asking a Sikh to remove his hat in public; asking a Muslim woman to remove her head scarf in public; having a Muslim male body searched by a woman officer). In general it is important for staff members to realise how their behaviour may inadvertently spark incidents with passengers from nationalities and cultures different than theirs.

Finally, the staff interviewed reported that another category of tensions concerned the interaction with privileged individuals, such as VIPs, politicians and high-ranking individuals. Some incidents have occurred in which some of these individuals behaved arrogantly towards staff, held unrealistic expectations about the level of service they could expect, or refused security checks and procedures (for instance because they did not want to be inspected by virtue of their social status). Also, our research participants reported at least one occurrence in which a politician on board a high-speed train used his authority to interfere with an on-going emergency operation.

\subsubsection{Poor communication due to language barriers}

Situations may occur in which staff and passengers are unable to understand each other due to language barriers. In these situations, the person is unable to articulate own thoughts either in the local language spoken by staff or in the relevant international language (e.g. English). This SCH may include different kinds of situations, such as, for instance, injured/sick passengers who are unable to report/communicate their own (deteriorating) health conditions; passengers unable to report a problem to staff (e.g. threat or hazard observed; missing relative); passengers misunderstanding staff instructions (e.g. not following the direction assigned by staff). Note that this hazard may be exacerbated when staff adopt poor communication strategies, such as repeating instructions or questions louder and louder, but without slowly articulating words - something that may trigger a defensive response of the concerned passenger, because of fear and/or embarrassment. 


\subsubsection{Ineffective reporting of security threats (relative to emergency prevention)}

Usually, passengers in transport hubs receive communications about the importance of noticing and reporting to authorities' items that may pose an immediate security threat, such as unattended packages, objects and baggage. However, our research participants reported cases in which passengers failed to report such threats effectively. For instance, passengers may fail to report a security threat, such as an unattended item, because they either are not aware of the threat, or may not perceive reporting it as an obligation. Furthermore, passengers may engage in an improper reporting procedure: they may themselves collect a potential threat so as to bring it to the police (instead of just calling the police). This latter case was reported relative to infrequent travellers, who, while attempting to do something useful, did not realise the potential danger they exposed themselves to. Finally, passengers may err on the cautious side, and over-report potentially security threats, which in fact turn out to be not dangerous. This latter behaviour may increase following the occurrence of a major disaster/terrorist attack, which usually enhances the level of alertness of the general public.

\subsubsection{Refusing to comply with staff safety instructions (during emergencies)}

During emergencies, passengers may fail to comply with staff instructions. For instance, passengers may act based on their own initiative. Unauthorised, self-initiated responses may be triggered by the increasing sense of danger, especially following a prolonged stay in a confined environment. For instance, in the case of the Norman Atlantic ferry disaster in 2015, some passengers abandoned the ship due to increased fear of the growing fire, and eventually died in deep waters. Again, in an incident mentioned during our interviews, a passenger on board a broken-down train, temporary stuck at a station, broke a window to get off without staff authorisation, and hence exposed himself to various hazards such as electrocution. These behaviours can arise as a consequence of a heightened sense of danger, combined with at least two culture-dependent factors such as low trust in authorities and a tendency for self-organisation (see e.g., $[17,18]$ ).

Also, familiarity with a particular transport hub may limit compliance with staff instructions, especially in emergencies. People have mental scripts for navigating through familiar environments and use these automatically without conscious thought $[19,20]$. Thus, regular users of a transport hub, such as commuters, may pay little attention to staff instructions, especially if these involve taking unfamiliar paths - such as taking elevators, stairs, or a tunnel to escape from a threat (e.g., fire) - and even if these are the quickest and safest ways to safety. This was exemplified in the case of the King's Cross underground station disaster in London in 1987, where the majority of the victims had taken the familiar route instead of the one instructed by the staff [19]. Overall, these considerations suggest that in transport terminals, familiar users such as commuters may require clear and targeted information to override their routine automatic routes, particularly in emergencies.

\subsection{Crowd-crowd interactions}

\subsubsection{Cultural conflicts between individuals/groups belonging to different cultural groups}

SCHs may include tensions between passengers belonging to different cultural groups. For instance, in an incident reported on the news, an 81-year old woman on an El Al flight from New York to Tel Aviv was invited to change seat, after an ultra-orthodox Jew refused to sit next to a woman [21]. The incident resulted in the woman concerned suing the airline. It seemed that the incident was not a single instance and others were reported to have occurred due to sexual discrimination by religious ultra-orthodox Jewish men, eventually causing 
flight delays [21, 22]. Other examples mentioned by the staff interviewed concerned potential conflicts between supporters of different football teams in the same terminal, e.g. train station airport. Although cultural difference, per se, is not necessarily the primary cause of conflict between groups, culture influences significantly the perceptions according to which cross-cultural conflict is formed [23].

\subsubsection{Fighting/revolting for resources (food, space, emergency exists, etc.)}

The literature on evacuation and crowd disasters has acknowledged the tension existing between the competitive and collaborative behaviour of passengers in emergencies [24, 25], and the fact that passengers may positively engage in collaborative behaviour in emergencies, for instance by keeping calm and supporting others. However, although less desirable, competitive behaviour may still occur. Passengers may fight for safety equipment during emergencies, and may push or even beat other passengers while attempting to evacuate quicker [26, 27]. In one of our interviews, a high-speed train steward reported an incident in which angry and hungry passengers on board a broken-down train revolted against him and other on-board staff in order to find food in the galley.

\subsubsection{Crowd-environment interactionPassengers entering restricted areas}

This category of SCHs involves passengers entering restricted areas, either inadvertently or intentionally. Inadvertent entrance into restricted areas may occur as wayfinding in transport hubs can be very demanding, especially for unfamiliar users or infrequent travellers. A serious wayfinding failure involves passengers inadvertently entering restricted security areas, something that may trigger an alarm and result in the evacuation of the hub (or part of it). For instance, on May 2016, at Cologne airport, a 62-year-old Spanish passenger rushing to his aircraft inadvertently entered a restricted area of Terminal One [28]. The event triggered a preventive evacuation of the terminal, while police officers searched for explosives, and several aircrafts were delayed. A similar security breach in the same airport with similar consequences involved a woman who was able to walk through the security screening area without being checked [29]. Besides age, familiarity and travel experience, another factor that may lead to this SCH is the lack of understanding of symbols across cultures: as Symonds succinctly puts it: "people of China does not [necessarily] understand the same symbols as American tourists" [30]. Corroborating this point is the observation of one of our research participants, a railway police director, who noted that asylum seekers in railways may not necessarily be familiar with basic symbols, such as the yellow line next to the rail.

Finally, sometimes passengers intentionally enter into restricted areas, such as rail tracks. These may be commuters in a hurry, or immigrants who do not perceive the danger of crossing tracks at railways station.

\subsubsection{Unintended misuse of (safety) equipment}

This behaviour involves the unauthorised and unjustified use of equipment by passengers who are unaware of the consequences or gravity of their actions. For instance, the railways operators we interviewed reported cases of improper emergency brake activation occurring when people accompanying passengers on board a high-speed train realised that the train was leaving the station while they were still on board. When approached by the train steward, the people concerned were surprised by the expensive penalty they had to pay. Analogous cases have been reported in the airport domain, in which the evacuation slide was unnecessarily deployed following the improper opening of a plane door by a first-time flyer, because of reasons such as mistaking an emergency exit for the toilet [31], or attempting to get fresh air 
before take-off [32]. Overall, the common factor across these types of behaviours is the lack of awareness of the consequences, such as delays and potential for injuries, arguably due to poor education and lack of familiarity with modern transport systems or a combination of both.

\subsubsection{Slow passenger mobilisation during emergencies}

While a quick mobilisation of passengers of a transport hub may be needed in certain situations, e.g. evacuation, there are factors that may hamper this process. First, passengers may react differently to public alarms in transport terminals, depending on the level of risk they perceive. This may vary according to different socio-demographic parameters, such as gender and age. Men and younger people are generally less risk-adverse than women and older people as documented by $[33,34]$. Thus, front-end operators have to consider that passengers might be more or less willing to evacuate according to their level of risk perception. Second, different passenger crowds may move at different speeds. Crowd walking speed might vary depending on several socio-demographic parameters, such as gender, age and group formation [35]. For instance, men walk faster than women, and adults and children walk faster than the elderly. People also walk more slowly when they are with others than when they are alone. Furthermore, as reported by [36], dress length also has a direct influence on people walking speed. These findings suggest that transport hub operators should be aware that crowds should not be treated as a collection of similar individuals.

\subsubsection{Misuse of social media information during emergencies}

Our research participants stressed that during an emergency, users of the transport hub may publish incorrect, partial, or incomplete information on social media, which may generate unjustified panic. Furthermore, young people sometimes record the emergency situation on their mobile phones instead of escaping from the danger and evacuating safely. This finding is indeed supported by a video analysis of Robinson and Cichomska (available in [37]) about the terrorist attack at Brussels airport.

\section{DISCUSSION AND CONCLUSIONS SUMMARY}

This study has identified 10 socio-cultural hazards (SCHs) that may occur in transport hubs. SCHs correspond to behaviours of members of the public in transit (and staff) that are dysfunctional to the efficiency, safety and quality of operations, and that therefore need to be addressed by front-end operators. SCHs may occur both during normal situations and during emergencies. In the former case, they may trigger incidents; in the latter case, they may add to the chaos and uncertainty of emergency operations, thus increasing their negative consequences. The framework of SCHs provides a means for front-end operators to identify and discuss issues they encounter in their interactions with the public.

The SCHs identified were formed into a framework comprising three high-level areas crowd-staff, crowd-crowd, and crowd-environment interactions. These three areas capture the fact that SCHS in transport hubs can be found in the interaction between individuals or groups in the crowd and the other elements of the surrounding context: staff members, other crowd members, and the information and physical environment.

\subsection{Practical value of the framework}

The ECCS framework has potential pragmatic value. It can enhance current transport hub safety and security risk assessment by prompting analysts to consider relevant categories of 
behavioural hazards that can impact substantially the risk profile of a given hub - either because they can trigger a crisis, or because they can greatly influence the consequences associated with an existing one. Also, the framework can become part of training packages for front-end operators of transport hubs. The framework can be used to sensitise operators regarding potential hazardous behaviours that may arise from the public in transport hubs, and their potential consequences in both normal and emergency situations. Indeed, the effective mitigation of $\mathrm{SCH}$ requires awareness and anticipation of them by front-end operators, as well as the use of adequate socio-cultural mitigations. To this end, the research team is currently developing a training package that aims to increase operators' intercultural skills, cross-cultural sensitivity, ability to cope with cultural differences, and awareness of cross-cultural differences in crowd behaviours as well as verbal and non-verbal communication skills, both one-to-one and one-to-many. Such skills are not necessarily covered by existing mandatory training programmes. Usually, these tend to cover the technical, procedural and regulatory aspects of the work, while providing limited coverage of the potential issues that may arise when interacting with members of the public. Yet, knowledge of these SCHs is part of the background of the more senior staff members [4], and is essential to contribute to safe and effective task performance both during normal and emergency situations.

Overall, the methodological approach taken ensured that the SCHs identified by the study were heavily based on the accounts of relevant research participants. As discussed in Section 2 , these included both back-end as well as front-end roles from the airport, railways, underground and maritime domains. This provided a relevant knowledge base which was triangulated with relevant academic literature and incidents reported in the media. Furthermore, an initial corroboration was obtained from a small-sample of four airport staff. Despite this, the framework can benefit from at least two types of future studies: (i) studies that will seek further corroboratory feedback from experts of other transport domains about dimensions such as the relevance, completeness, and correctness of the ECCS framework; and (ii) studies that will deepen the understanding of viewpoints of members of the public involved in SCHs, for instance through in-depth interviews and ethnographic observations of passengers in transport hubs. In turn, these efforts are expected to increase the validity and the generalisability of the framework.

Finally, note that, while the framework has essentially stressed the undesirable behaviours that may arise from the interaction of the public and the transport hub, previous research indicates that members of the public may also play a positive role in emergency management. Therefore, it is anticipated that the public may be considered a resource in the development of the socio-cultural mitigations. Thus, the authors advocate that the ECCS framework be extended or complemented with an analogous taxonomy of the positive behaviours that can be played by the public in transport hubs, in normal as well as in emergency situations.

\section{ACKNOWLEDGEMENTS}

This work is supported by funding from the European Commission through the IMPACT project (H2020 GA no. 653383). The authors wish to express their gratitude to the project coordinator, Dr Alessandra Tedeschi, for her support to this study, and to the members of the IMPACT External Stakeholder Group and the front-end staff members involved in the study.

\section{REFERENCES}

[1] Skybrary, 'Unruly Passengers - SKYbrary Aviation Safety', 2016, available at http:// www.skybrary.aero/index.php/Unruly_Passengers\#The_Problem (accessed 30 January 2017). 
[2] ICAO, Annex 17: Security. Safeguarding International Civil Aviation Against Acts of Unlawful Interference, 9th edn., International Civil Aviation Organisation, 2011.

[3] IATA, Guidance on unruly passenger behaviour and management. International Air Transport Association, 2012.

[4] Cheng-Hua, Y. \& Hsin-Li, C., Exploring the perceived competence of airport ground staff in dealing with unruly passenger behaviors. Tourism Management, 33(3), pp. 611$621,2012$.

https://doi.org/10.1016/j.tourman.2011.07.001

[5] Hella, J., Cabin violence: assessment and management. Cabin Safety Update, 7(9), pp. 28-31, 2001.

[6] ICAO, Doc 9811 Manual on the Implementation of the Security Provisions of Annex 6. International Civil Aviation Organisation, 2000.

[7] Kulatunga, U., Impact of culture towards disaster risk reduction. International Journal of Strategic Property Management, 4, pp. 304-313, 2010.

https://doi.org/10.3846/ijspm.2010.23

[8] Arunotai, N., Saved by an old legend and a keen observation: The case of Moken sea nomads in Thailand. Indigenous Knowledge for Disaster Risk Reduction, 73, 2008.

[9] Lavigne, F., De Coster, B., Juvin, N., Flohic, F., Gaillard, J.C., Texier, P., Morin, J. \& Sartohadi, J., People's behaviour in the face of volcanic hazards: Perspectives from Javanese communities, Indonesia. Journal of Volcanology and Geothermal Research, 172(3), pp. 273-287, 2008.

https://doi.org/10.1016/j.jvolgeores.2007.12.013

[10] Schreier, M., Qualitative Content Analysis in Practice, Thousand Oaks, California: SAGE Publications, 2012.

[11] Ritchie, J. \& Spencer, L., Qualitative data analysis for applied policy research. In The Qualitative Researcher's Companion, eds A.M. Huberman \& M.B. Miles, Thousand Oaks, California, USA: SAGE Publications, pp. 305-329, 2002.

[12] Smedley, B.D., Stith, A.Y. \& Nelson, A.R., Unequal treatment: Confronting racial and ethnic disparities in health care (full printed version), National Academies Press, 2002.

[13] Devine, P.G., Stereotypes and prejudice: Their automatic and controlled components. Journal of Personality and Social Psychology, 56(1), pp. 5-18, 1989.

https://doi.org/10.1037/0022-3514.56.1.5

[14] Van Ryn, M. \& Fu, S.S., Paved with good intentions: do public health and human service providers contribute to racial/ethnic disparities in health?. American Journal of Public Health, 93(2), pp. 248-255, 2003. https://doi.org/10.2105/AJPH.93.2.248

[15] Jones, A., Concept paper: Cultural competence: Solutions and strategies for emergency medical services, 2007.

[16] Sutherland, D., The role of homeland security and training. In The Arab-American Handbook: A Guide to the Arab, Arab-American, and Muslim Worlds, 2nd edn., ed. N. Shora, Cune Press, 2009.

[17] Hofstede, G.H., Culture's Consequences: Comparing Values, Behaviors, Institutions and Organizations Across Nations, Sage, 2001.

[18] Welzel, C., How selfish are self-expression values? A civicness test. Journal of CrossCultural Psychology, 41(2), pp. 152-174, 2010.

https://doi.org/10.1177/0022022109354378 
[19] Donald, I. \& Canter, D., Intentionality and fatality during the King's Cross underground fire. European Journal of Social Psychology, 22(3), pp. 203-218, 1992. https://doi.org/10.1002/ejsp.2420220302

[20] Charlton, S.G. \& Starkey, N.J., Driving without awareness: The effects of practice and automaticity on attention and driving, 2011.

[21] Sherwood, H., Retired lawyer sues Israeli airline after she was asked to move seat. The Guardian, 01 April 2016.

[22] Paulson, M., When a plane seat next to a woman is against orthodox faith. The New York Times, 09-April-2015.

[23] Avruch, K., Cross-cultural conflict. Conflict Resolution, 1, pp. 45-57, 2009.

[24] Chertkoff, J.M. \& Kushigian, R.H., Don't Panic: The Psychology of Emergency Egress and Ingress, Praeger Publishers, 1999.

[25] Rogsch, C., Schreckenberg, M., Tribble, E., Klingsch, W. \& Kretz, T., Was it panic? An overview about mass-emergencies and their origins all over the world for recent years. In Pedestrian and Evacuation Dynamics 2008, Springer, pp. 743-755, 2010. https://doi.org/10.1007/978-3-642-04504-2_72

[26] Sabin, L., Italy ferry fire: Rescued passengers describe violence and panic on board stricken vessel, available at http://www.independent.co.uk. [Online], available at http:// www.independent.co.uk/news/world/europe/norman-atlantic-ferry-fire-two-britishmen-still-not-rescued-from-burning-italian-ship-9947674.html (accessed 07 Febuary 2017).

[27] Rossington, B., Men HIT women in fight to rescue helicopters during Italy ferry fire. Mirror, 30 December 2014. Available at http://www.mirror.co.uk/news/world-news/ italy-ferry-fire-men-hit-4893979. (accessed 16 September 2016).

[28] Confused Spaniard to blame for alarm at Cologne airport. The Local, 30-May-2016. Available at: http://www.thelocal.de/20160530/breaking-flights-stopped-over-securityalert-at-cologne-airport. (accessed 31 August 2016).

[29] Withnall, A., Cologne airport Terminal 2 evacuated due to security breach | The Independent, available at http://www.independent.co.uk, 01-Mar-2016. [Online], available at http://www.independent.co.uk/news/world/europe/cologne-airport-evacuated-dueto-security-breach-a6904766.html (accessed 07 Febuary 2017).

[30] Symonds, P., The Complexities of Human Wayfinding in Airports, 2014.

[31] Branigan, T., Chinese passenger opens plane door for fresh air. The Guardian, 18 Dec 2014, [Online], available at https://www.theguardian.com/world/2014/dec/18/chinesepassenger-opens-plane-door-for-fresh-air (accessed 12 July 2016).

[32] Kitching, C., Flyer mistakes emergency exit for loo and triggers inflatable slide, Mail Online, 27 March 2016. Available at http://www.dailymail.co.uk/travel/travel_news/ article-3511283/China-Southern-Airlines-passenger-opens-emergency-exit.html (accessed 12 July 2016).

[33] Byrnes, J.P., Miller, D.C. \& Schafer, W.D., Gender Differences in Risk Taking: A MetaAnalysis, American Psychological Association, 1999.

[34] Deakin, J., Aitken, M., Robbins, T. \& Sahakian, B.J., Risk taking during decisionmaking in normal volunteers changes with age. Journal of the International Neuropsychological Society, 10(4), pp. 590-598, 2004. https://doi.org/10.1017/S1355617704104104 
[35] Willis, A., Gjersoe, N., Havard, C., Kerridge, J. \& Kukla, R., Human movement behaviour in urban spaces: Implications for the design and modelling of effective pedestrian environments. Environment and Planning B: Planningand Design, 31(6), pp. 805-828, 2004

https://doi.org/10.1068/b3060

[36] Almejmaj, M., Meacham, B. \& Skorinko, J., The effects of cultural differences between the west and Saudi Arabia on emergency evacuation-clothing effects on walking speed. Fire and Materials, 39(4), pp. 353-370, 2015. https://doi.org/10.1002/fam.2227

[37] Cichomska, K., et al., D1.3: Theoretical Framework of Cross-Cultural and PsychoSocial Crowd Behaviour and Management in Transport Terminals. IMPACT Deliverable D1.3, January 2016. 